\title{
Musik und Medizin
}

Das vorliegende Themenheft beschäftigt sich mit dem Spannungsfeld von Musik und Medizin. Damit betreten wir, zumindest für die Nervenheilkunde, Neuland. Zwar hat es in der Vergangenheit unzählige Übersichten und medizinische Veröffentlichungen zu diesem Thema gegeben, die meisten mussten jedoch in die Kategorie feuilletonistischer, zum Teil sogar romantisierender Verklärungen dieses Gebiets kategorisiert werden. Unser Ansatz war es, verschiedene Themen aus dem Bereich der Nervenheilkunden unter der Überschrift „Musik und Medizin“ zusammenzubringen, um eine vielfältige moderne und auch klinisch orientierte Forschungslandschaft zu beschreiben, die nicht nur die segensreichen und nützlichen Aspekte dieses Spannungsfelds beschreibt, sondern auch dessen Limitierungen. Wir wollen somit einen Beitrag zu der wissenschaftlichen Diskussion über die vielfältigen Überschneidungen von Musik und Medizin leisten.

Im Beitrag von Stefan Evers geht es auf einer Meta-Ebene genau um dieses Spannungsfeld, indem eine Systematik der verschiedenen Überschneidungsebenen vorgeschlagen wird. Dies geschieht ausdrücklich aus wissenschaftlicher Sicht, d. h. Medizin und Musik(wissenschaft) werden als eigenständige Forschungsgebiete gesehen, um dann die interdisziplinären Forschungsobjekte zu beschreiben. Dabei gehört - wie überall in der klinischen Medizin - auch die Patientenorientierung mit in diese Systematik.

Die Bedeutung der Musikerziehung in Kindergarten und Schule ist an sich unumstritten. Dennoch nimmt das gemeinsame Musizieren in beiden Einrichtungen eher $\mathrm{ab}$ als zu. In einer Übersicht stellen Thomas Kammer und Manfred Spitzer Ergebnisse von empirischen Studien dar, die experimentell die positive Wirkung von aktivem Musizieren auf Sprachentwicklung, exekutive Funktionen, sowie allgemeine Intelligenz und soziale und affektive Fähigkeiten nachweisen. Diese sogenannten Transfereffek- te können den kulturell unumstrittenen Stellenwert der Musikerziehung noch untermauern.

Claudia Spahn beleuchtet die spezifischen psychosomatischen Beschwerdebilder, die bei Berufsmusikern auftreten können. Sie hebt zwei Krankheitsbilder hervor, die gehäuft beobachtet werden: Auftrittsangst und somatoforme Schmerzstörung. Die Auftrittsangst kann als Lampenfieber die Musiker beflügeln. Sie kann in gravierenderer Form auch beeinträchtigen und bis zur Berufsunfähigkeit führen. Bei beiden Krankheitsbildern gibt es spezifische therapeutische Empfehlungen.

In dem Beitrag von Rosemarie Tüpker geht es um die Musiktherapie „an sich“. Hier wird deutlich, dass die therapeutische Anwendung von Musik in der Medizin eine eigenständige Therapiedisziplin darstellen kann und dann in das Repertoire der psychotherapeutischen Verfahren gehört, es geht hier also um eine Therapeutenbeziehung. Dagegen wird in dem Beitrag von Florina Speth der mehr "medizinische“ Einsatz von Musik in der Therapie beleuchtet. Hier sind zahlreiche Studien in den letzten Jahren erschienen, die insbesondere die Bedeutung der Musik in der neurologischen Rehabilitation belegen. Hier kommt „Musiktherapie" ohne therapeutische Beziehung aus, sollte aber deswegen nicht weniger individuell und evidenzbasiert angewendet werden.

In dem Beitrag von Ralph Spintge geht es ebenfalls um eine spezifische therapeutische Wirkung von Musik, nämlich in der Schmerztherapie. Hier überschneiden sich exemplarisch die Musiktherapie als eigenständiges therapeutisches Verfahren mit einer therapeutischen Beziehung und der Einsatz von Musik z. B. in der Analgesie bei Operationen, d. h. also der mehr mechanistische Einsatz von Musik. Interessanterweise hat sich gerade in der Schmerzmusiktherapie der individualisierte (englisch auch tailored genannte) Einsatz von Musik in der Therapie weit entwickelt.

Im Editorial beleuchtet Manfred Spitzer den Diskussionsstand zur evolutionä-

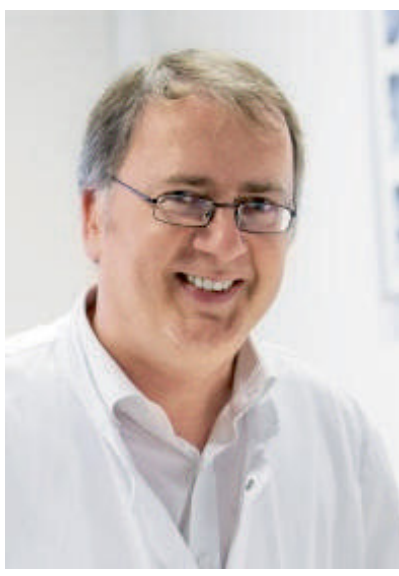

Prof. Dr. med. Dr. phil. Stefan Evers, Neurologische Klinik, Krankenhaus Lindenbrunn, Coppenbrügge

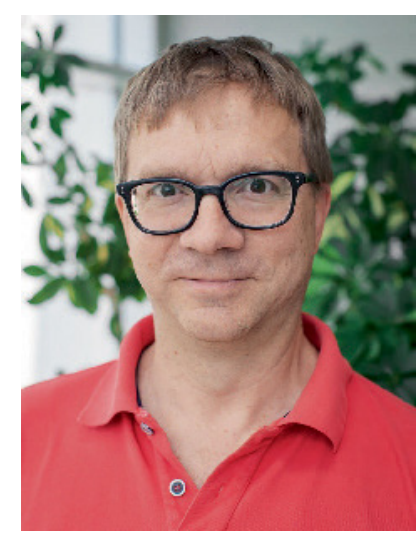

Prof. Dr. med. Thomas Kammer, Sektion für Neurostimulation, Klinik für Psychiatrie und Psychotherapie III, Universität UIm 
ren Rolle von Musik. Im Hinblick auf Anpassung und natürliche Selektion wurde sie zunächst als völliger Unsinn bzw. als Handicap, das besondere Fitness seines Trägers anzeigt betrachtet, dann als Teil des Paarungsverhaltens im Sinne der von Darwin später formulierten Idee der sexuellen Selektion, und schließlich dann doch wieder als Anpassung im Sinne der Selektion von Kooperation und Hilfsbereitschaft, also sozialen Verhaltensweisen, die für die Gesamtgruppe von großem Vorteil sind.
Auch die Rubrik „Geist und Gehirn" von Manfred Spitzer greift das Thema Musik auf. Und im Beitrag „Musik: von der Koordination zur Kooperation“" werden Studien zum koordinierten Gehen, Singen, Musizieren und Tanzen vorgestellt, die nicht nur miteinander erfolgenden Bewegungen (koordiniertes Verhalten) beleuchten, sondern zeigen, dass sich die abgestimmten Bewegungen auch auf die Abstimmung von Interessen und damit auf kooperatives Verhalten positiv auswirken. Diese Studien sind sowohl theoretisch als auch ganz praktisch von Bedeutung.
Jede Theorie der Evolution von $\mathrm{Mu}$ sik muss auch diese Daten in Betracht ziehen. Und jeder Praktiker im Bereich von Erziehung und Bildung auch!

Wir wünschen eine interessante Lektüre dieses Themenheftes und hoffen auf eine insgesamt größere Aufmerksamkeit für dieses interdisziplinäre Forschungs- und Anwendungsfeld.

\section{Stefan Evers, Coppenbrügge}

Thomas Kammer, Ulm 